\title{
Plagiarism again: Sreenivas and Srinivas, with an update on Marcu
}

\author{
Denis Bouyssou • Silvano Martello • \\ Frank Plastria
}

Published online: 7 March 2009

(C) Springer-Verlag 2009

\section{Introduction}

In recent years many scientific journals noticed an increasing number of submissions of plagiarized papers. The Editor-in-Chief of an important international journal, that receives very many submissions every year, recently privately wrote us "I had to reject on such basis over a dozen of submissions just this year". The smaller size of our journal limits the number of such situations, but does not eliminate them. We report here a few cases we encountered, in the hope that officially associating people with proved plagiarism cases can contribute to fight this highly unethical phenomenon. On the one hand articles of this kind make it easier to Editors of scientific journals to identify suspicious submissions by googling on the internet, and on the other hand we believe that they are a deterrent for (would be) plagiarists against future attempts.

D. Bouyssou

CNRS, LAMSADE, Université Paris Dauphine,

Place du Maréchal de Lattre de Tassigny, 75775 Paris Cedex 16, France

e-mail: 4or@lamsade.dauphine.fr

S. Martello $(\bowtie)$

DEIS, Università di Bologna, Viale Risorgimento 2, 40136 Bologna, Italy

e-mail: dipdeis.4or@unibo.it

F. Plastria

MOSI-Department of Mathematics, Operational Research,

Statistics and Information Systems, for Management,

Vrije Universiteit Brussel, Pleinlaan 2, 1050 Brussels, Belgium

e-mail: 4or.be@vub.ac.be 


\section{M. Sreenivas and T. Srinivas}

In October 2007 M. Sreenivas and T. Srinivas submitted to 4OR the paper "The transportation: More-for-less criterion" (it can be downloaded from our web page: http://homepages.vub.ac.be/ fouror/). One of the referees who were asked to review it immediately answered the following:

I found something hidden in this paper! This is a paper of Storøy (2007). The text of the paper you sent to me is almost identical to the source on the web. I wonder whether we, as community, should do something about "authors" like Sreenivas and Srinivas.

We communicated to these "authors" our ban from publishing in $4 O R$, and our intention to publicize by all appropriate means the fact that they submitted such a paper. Their answer is worth reporting:

Dear Sir,

We are very sorry in sending such type of article to your journal. Actually, we are working on the topic. Sorry once again.

M. Sreenivas

Dr. T. Srinivas

(Is the second sentence a threat?)

We conducted additional investigations. Although the authors were expressing regret, this did not prevent them from submitting their plagiarized text to another well known OR Journal, namely the European Journal of Operational Research. Fortunately, the plagiarism was also detected there, and the results of our investigations, which we had reported on our web page http://homepages.vub.ac.be/ fouror/, turned out to be helpful in this process.

Furthermore, we discovered that Sreenivas and Srinivas were members of the repository of e-prints Optimization Online, which hosts the plagiarized paper, and deposited two other contributions. The first of these papers, http://www.optimization-online. org/DB_FILE/2007/09/1774.pdf, is dated September 2007, and is plagiarized from a 2005 paper by Colannino and Toussaint (2005). The second paper, http://www. optimization-online.org/DB_FILE/2007/10/1814.pdf, is dated October 2007, and is plagiarized from a 2005 paper by Tseng etal. (2005). The two plagiarized papers are online at our web page above.

It seems to us that the regret the two authors expressed was not really sincere, and that we are facing a case of professional plagiarism more or less like Dănuţ Marcu (see the next section).

\section{An update on Marcu}

In a previous editorial (Bouyssou et al. 2006) we reported on a plagiarized submission by Dănuţ Marcu from Bucharest. The submission and the plagiarized paper can be downloaded from our web page mentioned above. A thorough search on the Internet led to a series of interesting findings. The same "author" had succeeded in publishing plagiarized texts in other journals, such as, e.g., Studia Universitatis Babes-Bolyai 
Informatica, Computer Science Journal of Moldova and Journal of the Indian Institute of Science. The Graph Theory White Pages listed eighty papers published by Marcu in the period 1990-2003, and he was classified in 10th position in the list of the authors with most journal articles (currently his name appears to have been removed).

After the publication of Bouyssou et al. (2006) some new interesting facts occurred. A. Soifer, Editor of Geombinatorics, opened an investigation (reported in Soifer 2007) of Marcu's submissions to Geombinatorics. He first offered him an opportunity to rebut the accusations, and Marcu's answer is worth to be reported in its entirety:

Dear Professor Soifer:

I consider that this very strong attack against me has an answer related to my 10th position in the Graph Theory White Pages. For me, nothing is not new [sic], since years ago these stupid and ridiculous attacks began. If you will consult the following addresses, perhaps, you will understand:

http://profiles.yahoo.com/drmarcu

http://www1.cs.columbia.edu/ sanders/graphtheory/people/Marcu.D.html Anyway, you decide whatever. I would greatly appreciate receiving an answer from you, concerning my papers submitted to Geombinatorics.

Yours faithfully,

Dănuţ Marcu

(The Yahoo page mentioned above no longer exists. Marcu has now a page at Geocities, http://www.geocities.com/drmarcu/PP_DM.html, where you can read "I am the author of more than 400 papers on mathematics, teletraffic and computer science.")

Soifer therefore started his investigation, with the following results. Marcu had published four papers in Geombinatorics, and all turned out to "nearly word-for-word repeat" previously published papers. A fifth paper, just submitted to the journal, turned out to repeat, almost word-for-word, Problem 787, proposed in Kaczynski (1971), and both solutions, from the "Problems and Solutions" section (Gibbs and Breisch 1971) of Mathematics Magazine (note that the plagiarized author, Theodore Kaczynski, currently serving a life sentence in a US prison, is worldwide known as Unabomber!).

The "fame" of Dănuţ Marcu greatly increased after the publication of Bouyssou et al. (2006) and Soifer (2007). By googling on the internet one will easily find his Wikipedia entry, where such articles are referenced.

\section{Conclusion}

After the publication of our previous note (Bouyssou et al. 2006) and of the one by Soifer (2007), to our knowledge Marcu did not succeed in publishing plagiarized papers. It is our hope that the present article will also stop the plagiarizing activities of Sreenivas and Srinivas and, above all, give second thoughts to possible would be plagiarists. 


\section{References}

Bouyssou D, Martello S, Plastria F (2006) A case of plagiarism: Dănuţ Marcu. 4OR-A Q J Oper Res 4:11-13

Colannino J, Toussaint GT (2005) A faster algorithm for computing the link distance between two point sets on the real line. Technical Report SOCS-TR-2005.5. School of Computer Science, McGill University

Gibbs RA, Breisch RL (1971) A match stick problem (solution to problem 787). In: Horton RE (ed) Problems and solutions. Mathematics Magazine, vol 44, pp 294-296

Kaczynski TJ (1971) Problem 787. Math Mag 44:41

Soifer A (2007) The case of Dr. Dănuţ Marcu: serial plagiarism and signing false statements. Geombinatorics XVI:293-296

Storøy S (2007) The transportation paradox revisited. Optimization Online http://www.optimization-online. org/DB_FILE/2007/09/1763.pdf

Tseng Y-Y, Yue WL, Taylor MAP (2005) The role of transportation in the logistics chain. In: Satoh K (ed) Proceedings of the eastern Asia society for transportation studies, vol 5, pp 1657-1672 